\title{
COUPLED MULTIPHYSICS MODELING OF AORTIC DISSECTION
}

\author{
R. SCHUSSNIG ${ }^{1,2}$ and T. P. FRIES ${ }^{1,2}$ \\ ${ }^{1}$ Graz Center of Computational Engineering, Krenngasse 37/I, \\ 8010 Graz, schussnig@tugraz.at, gcce.tugraz.at \\ ${ }^{2}$ Institute of Structural Analysis, Graz University of Technology, \\ Lessingstraße 25/II, 8010 Graz, fries@ tugraz.at, ifb.tugraz.at
}

Key words: Aortic Dissection, Hyperelasticity, Fluid-Structure Interaction, Patient-Specific Simulation, Biomechanics, Multiphysics

\begin{abstract}
Computational modeling of the cardiovascular system plays an increasingly important role in biomedicine, as it allows for non-invasive investigations of the status-quo and studying the influence of different treatment options available. The goal is to incorporate patient-specific datasets to obtain socalled digital twins to increase relevance of virtual surgery and support clinical decision making. In this context, aortic dissection is particularly challenging, since the overall system behavior strongly depends on the interplay between tissue deformation and blood flow, giving rise to a fully coupled fluid-structure interaction problem. To account for the complex physics, several additional modeling aspects such as prestress, advanced constitutive models respecting fibre orientation and suitable boundary conditions for the fluid and solid phases have to be considered. Within this study, these special techniques are applied to a patient-specific dataset, for which first results are presented highlighting their relevance.
\end{abstract}

\section{INTRODUCTION}

Aortic dissection is a life-threatening condition, in which pathologically altered layers of the aorta rupture and thereby allow for blood flow between individual layers of the media. The propagation of this so-called false lumen is dominated by a complex interdependency of pulsating blood flow and tissue deformation, which are in turn highly influenced by the position of the primary entry tear and geometric configuration of the intimal flap. This part of the dissected aortic wall separates true and false lumina and thereby plays a major role in the onset of dissection and overall disease progression. Consequently, the multiple interacting fields composing the coupled problem must be considered to obtain results relevant for management and treatment decisions in clinical application.

Despite the remarkable advancements in the field of computational biomechanics and haemodynamics during the recent years (cf. for work related to aortic dissection [1-6]), numerical simulations of fluidstructure interaction in patient-specific cardiovascular settings remain challenging. Nonetheless, several advancements with regards to simulating aortic dissection with fluid-structure interaction are reported in literature.

One of the first studies on computational modeling of aortic dissection by Qiao et al. [7] investigated the effects of bypassing a DeBakey III aortic dissection, comparing the haemodynamic parameters and intimal flap displacement before and after virtual surgical intervention using two alternative bypass strate- 
gies. The geometry was reconstructed using MRI data, yet simplified by excluding any branches and extruding uniformly by $2 \mathrm{~mm}$ to generate the solid domain. Blood was considered as a Newtonian fluid with parameters chosen in the physiological range, while a linear-elastic solid with an increased density of $2000 \mathrm{~kg} / \mathrm{m}^{3}$ and Young's modulus of $800 \mathrm{kPa}$ was employed. As for boundary conditions, realistic inflow conditions were considered together with a reference pressure at the outlet, neglecting external tissue response and intramural pressure. Flap displacements at peak systole of $1.3 \mathrm{~mm}$ were computed and compared to a rigid wall simulation, yielding significant differences in the fluid pressure and velocities observed.

Another study was conducted by Alimohammadi et al. [8]. Therein, the patient-specific lumen geometry of a 54 year old female was reconstructed from CT images and extended by a fixed $2.5 \mathrm{~mm}$ to obtain a suitable geometric representation of the vessel wall. Blood flow was modeled combining the Carreau-Yasuda rheological law with a shear-stress transport turbulence model, while Windkessel outlet parameters were fit to match patient data and realistic inlet velocity profiles were prescribed. Regarding the aortic tissue, an isotropic, incompressible, hyperelastic response of the tissue was chosen. The center points of the outlets were fixed in the global horizontal directions and a uniform external pressure of $52.5 \mathrm{mmHg}$ was applied, neglecting the elastic response of the surrounding tissue. Large differences in flow distribution and oscillatory shear index (OSI) were reported, even though only small deformations of the intimal flap $>0.8 \mathrm{~mm}$ were observed. Limited displacements of the false lumen might be caused by the high shear-modulus of $360 \mathrm{kPa}$ selected or simply result from the specific geometry. Nonetheless, the authors state that critical differences and key features might not be captured accurately enough, when neglecting the wall and flap movement in the modeling of aortic dissection.

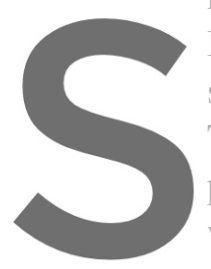

In an attempt to conside

structure interaction prob

Therein, the vessel wall

pressure. The intimal flap,

was considered under laminar flow
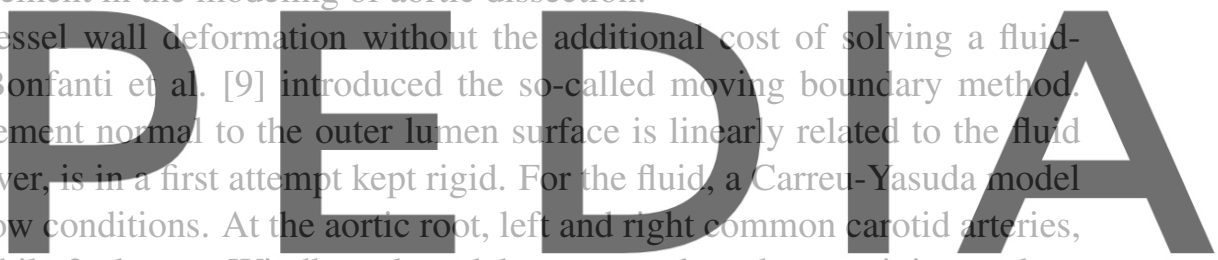

flow profiles were prescribed, while 3-element Windkessel models were used on the remaining outlets.

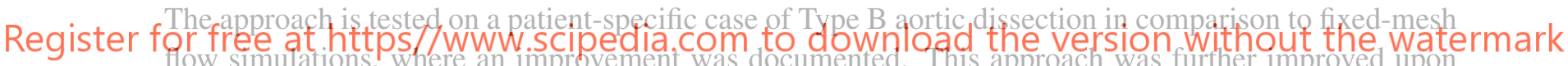

in $[10,11]$, introducing a deformable intimal flap. In analogy to the basic method, a membrane of zero

thickness is introduced, which deforms according to the same law as points on the external boundary, i.e., dependent solely on the fluid pressure (and tuning parameters). Comparing full fluid-structure interaction, fixed-wall flow simulations and the newly introduced method, pressure and flow rates of the moving boundary method were closer to results obtained with fluid-structure interaction than the fluid flow in a fixed domain.

Qiao et al. [12] presented the first study of aortic dissection which considered for fluid-structure interaction with a two-phase non-Newtonian fluid model. A patient specific geometry was reconstructed from CT images and a uniform wall thickness of $2 \mathrm{~mm}$ prescribed. Three-element Windkessel models were applied at the outlets together with an inlet volumetric flow rate with parameters taken from literature. The tissue was assumed to feature isotropic, linear elastic material behaviour and assigned an increased density of $2000 \mathrm{~kg} / \mathrm{m}^{3}$. The flap displacement at peak systole of $\leq 1 \mathrm{~mm}$ remained below the range to be expected under physiological conditions. Nonetheless, important conclusions can be drawn from this study with focus on haemodynamic parameters, since the multi-phase nature of blood is accounted for, which consequently results in the main subject of investigation being the comparison of OSI and time- 
averaged wall shear stress (TAWSS) as well as the deformation of the vessel wall during a cardiac cycle with fixed-wall and single-phase non-Newtonian models. Relative differences in the wall shear stress of up to $\pm 50 \%$ during the cardiac cycle between single-phase and two-phase non-Newtonian models highlight potential for improving the modeling of blood flow and thrombus formation in the setting of aortic dissection.

In [13], fluid-structure interaction simulations of aortic dissection were performed modeling blood as an incompressible Newtonian fluid and the tissue as an isotropic, hyperelastic and quasi-incompressible continuum. Patient-specific 4D MRI data is used to prescribe a parabolic velocity profile at the inlet, matching measured volumetric flow rates and fit the parameters of three-element Windkessel models. Also, Robin boundary conditions were included to account for external tissue support. A prestressing algorithm was applied to compute a prestress-tensor $\mathbf{S}_{0}$ in the solid domain except for the flap, which is assumed to be prestress-free in diastole. The anatomic model is generated from high resolution CTA data aquired in mid-diastole and further processed manually, i.e., smoothed and extended, to obtain a fixed wall and flap thickness of $2 \mathrm{~mm}$. Flow rates, pressure differences and TAWSS in true and false lumen and geometric quantities, such as cross-sectional area or diameter change, are reported. That contribution highlights the importance of flap stiffness by varying the elastic modulus of the flap region between $20 \mathrm{kPa}$ and $800 \mathrm{kPa}$ and validates computational results by comparing them with clinical data, showing good agreement in large parts including the noteworthy large flap motion within the realistic range of up to $13.4 \mathrm{~mm}$ for some parameter choices considered. The combination of an adequate geometric approximation, a monolithic fluid-structure interaction solver including realistic boundary conditions for both fluid and solid sub-problems with patient-specific clinical datasets leads to one of the most holistic

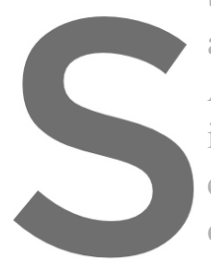
approaches presented up to date All of these studies underline the important in aortic dissect consequently greatly affect obtained results and the ga
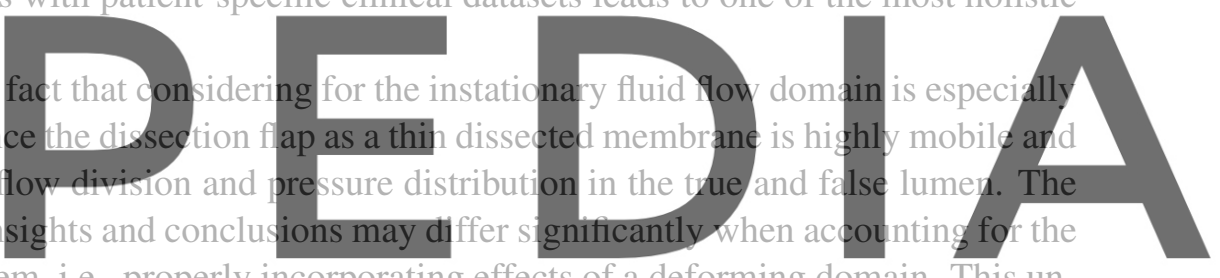

fully coupled multiphysics problem, i.e., properly incorporating effects of a deforming domain. This un-

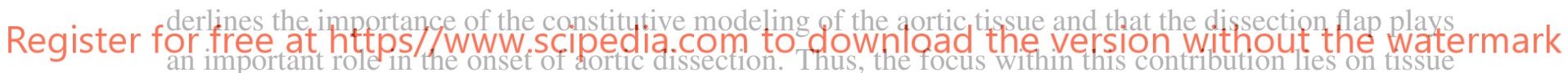

mechanics to better predict vessel wall displacements and stress concentrations. A fibre-reinforced, incompressible hyperelastic continuum is considered [14], accounting for the highly nonlinear anisotropic response of collagen fibres. Consequently, the orientation of fibres in the reconstructed geometry plays a crucial role with respect to the overall system response. However, the dissection flap characteristic for aortic dissection complicates the construction of local coordinate systems relevant for the fibre directions. A new hybrid technique combining common approaches based on the solution of a simple boundary value problem and geometric considerations result in a natural fibre orientation. Together with an algorithm to incorporate effects of prestress present in the tissue at time of image acquisition, the presented methodology leads to a fully coupled fluid-structure interaction simulation of aortic dissection featuring suitable constitutive models.

\section{MODELING ASPECTS}

The modeling and simulation of patient-specific aortic dissection requires including several non-standard modeling techniques, developed during the past decade of advancement in computational haemodynam- 
ics. Those well-established techniques need to be adapted to the present case, in order to, e.g., account for fibre-reinforcement and its orientation in the tissue or prestress at image acquisition. The following sections introduce the mechanical models and comment on their peculiarities.

\subsection{Balance equations}

Blood is considered an incompressible Newtonian fluid, which is a reasonable assumption balancing computational cost and accuracy made in line with numerous studies in literature [1,6]. Therefore, balance of linear momentum and continuity equation are expressed using the fluid velocity $\mathbf{v}$ and pressure $p$ as primary variables in Arbitrary-Lagrangian-Eulerian (ALE) formulation, posed in the moving domain $\Omega_{f}(t)$ as

$$
\left.\begin{array}{rl}
\rho_{\mathrm{f}}\left[\hat{\partial}_{t} \mathbf{v}+\nabla \mathbf{v}(\mathbf{v}-\hat{\mathbf{v}})\right]-\nabla \cdot \sigma_{\mathrm{f}}=\mathbf{0} \\
\nabla \cdot \mathrm{v}=0
\end{array}\right\} \text { in } \Omega_{\mathrm{f}}(t) \times(0, T],
$$

with fluid density $\rho_{\mathrm{f}}, \hat{\partial}_{\mathrm{t}} \mathbf{v}$ denoting the ALE time-derivative of functions defined in the fluid domain moving with velocity $\hat{\mathbf{v}}$ and fluid's Cauchy stress tensor defined for Newtonian fluids as

$$
\sigma_{\mathrm{f}}=-p \mathbf{I}+\mu_{\mathrm{f}}\left[\nabla \mathbf{v}+(\nabla \mathbf{v})^{\mathrm{T}}\right]
$$

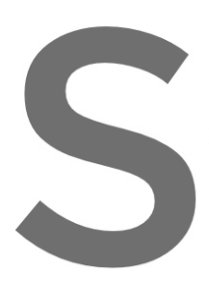

with dynamic viscosity

The focus of this study reinforced (quasi-) inco

introduce the displacemen
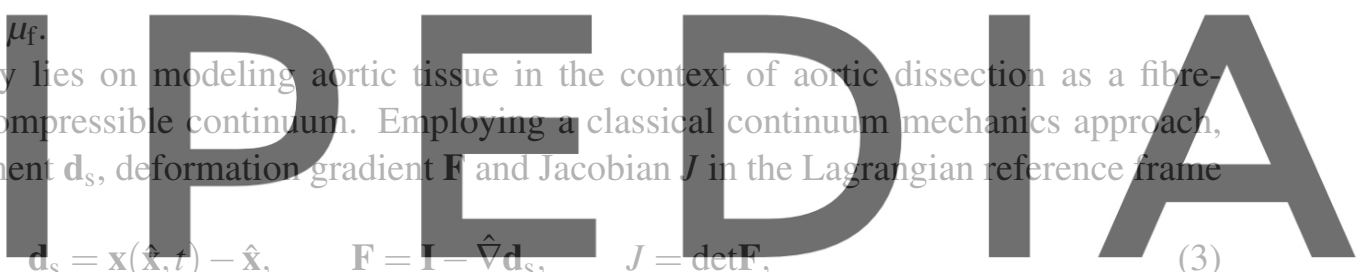

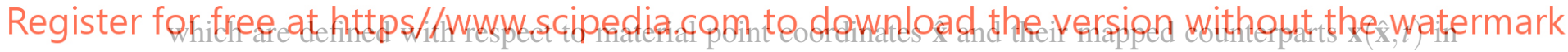

the current configuration. This allows writing linear momentum balance in the solid sub-domain in terms

of displacement from reference to current configuration only

$$
\rho_{\mathrm{s}} \frac{\partial^{2}}{\partial t^{2}} \mathbf{d}_{\mathrm{s}}-\hat{\nabla} \cdot \mathbf{P}=\mathbf{0} \text { in } \hat{\Omega}_{\mathrm{s}} \times(0, T]
$$

denoting by $\rho_{\mathrm{s}}$ the solid density and introducing $\mathbf{P}$, the first Piola-Kirchhoff stress tensor. Further, the right Cauchy-Green strain tensor $\mathbf{C}=\mathbf{F}^{\mathrm{T}} \mathbf{F}$ enables writing the constitutive relation based on the strain energy density $\Psi$ defined according to [14]

$$
\Psi=\frac{\mu_{\mathrm{s}}}{2}\left(\bar{I}_{1}-3\right)+\frac{\kappa_{\mathrm{b}}}{4}\left(J^{2}-1-\ln J\right)+\frac{k_{1}}{2 k_{2}} \sum_{i=4,6}\left[\exp \left\{k_{2}\left[\kappa \bar{I}_{1}+(1-3 \kappa) \bar{I}_{i}-1\right]^{2}\right\}-1\right]
$$

using the invariants

$$
\bar{I}_{1}=J^{-2 / 3} \mathbf{C}: \mathbf{I} \quad \text { and } \quad \bar{I}_{i}=J^{-2 / 3} \mathbf{C}:\left(\mathbf{M}_{i} \otimes \mathbf{M}_{i}\right), \quad i=4,6,
$$


and symmetric mean fibre directions $\mathbf{M}_{i}=[ \pm \sin \alpha, \cos \alpha, 0]^{\mathrm{T}}$. Then, the system is finally closed via

$$
\mathbf{P}=\mathbf{F S} \quad \text { and } \quad \mathbf{S}=2 \frac{\partial \Psi}{\partial \mathbf{C}} .
$$

The contributions to the strain energy density (5) are of neo-Hookean type, combined with an incompressibility penalization and the highly anisotropic, nonlinear contribution of two symmetrically dispersed fibre families. Such complex constitutive modeling of aortic tissue has not yet been applied in the context of patient-specific aortic dissection, but is expected to have a major influence on displacements and stresses and therefore on the overall solution due to the large strains encountered in the present setting.

\subsection{Mesh construction}

Geometry acquisition and constructing suitable discrete representations of the computational domain are particularly cumbersome in the context of aortic dissection. Compared to standard lumen reconstruction, the topology is found to be far more complex due to the thin dissection membrane separating true and false lumina. The starting point for the presented finite element mesh is the fluid-structure interface as a surface mesh in three-dimensional space thankfully provided by [13]. This surface mesh is based on CTA scans of chest and abdomen of a 52-year old man with a residual Type B aortic dissection, resulting in a voxel dataset of $512 \times 513 \times 1027$, at a resolution of $0.63 \mathrm{~mm} \times 0.63 \mathrm{~mm} \times 0.70 \mathrm{~mm}$. The proximal intimal tear is located at the left subclavian artery and extends into both left and right iliac arteries, where re-entry tears are present. Qnly the left subclavian artery is supplied by true and false
lumina, while the remaining branch vessels are supplied by the true lumen only. The whole aortic ree
is considered as depicted in Figure 1 (right). since cutting the true and false lumina would neces sitate
additional assumptions regarding flow rates to construct suitable boundary conditions, thereby further
increasing complexity and decreasing stability. A uniform wall-thickness of $2 \mathrm{~mm}$ is assumed, mapping points into averaged face-normal direction via

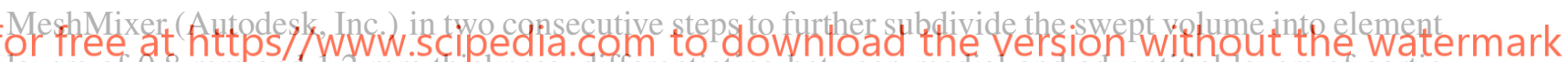
layers of $8.8 \mathrm{~mm}$ and $1.2 \mathrm{~mm}$ thickness, differentiating between medial and adventitial layers of aortic tissue. The resulting volumetric mesh is shown in Figure 1 (left), indicating distinct tissue layers and fluid volume by element colors. Finally, the volumetric elements in the flap region are identified, which (i) have nodes on the fluid-structure interface and (ii) are not in the layer of $n$-th neighbors of elements touching the external boundary, with $n$ set accordingly.

\subsection{Fibre orientation}

Collagen fibres are main contributors to the overall stiffness of aortic tissue $[5,15]$ and thus heavily influencing vessel wall deformation in response to haemodynamic forces acting on the fluid-structure interface. However, representing the natural fibre distribution in a given patient-specific geometry is non-trivial owing to the complex topology. Standard techniques solely based on the Laplace operator fail quickly, as one would need to prescribe suitable boundary conditions also in the flap region. Constructing meaningful local coordinate systems to orient the mean fibre directions is therefore done in a hybrid way, combining approaches using weak solutions to a stationary and easily solvable boundary value problem based on the Laplace equation with purely geometric considerations, as described in the following steps: 

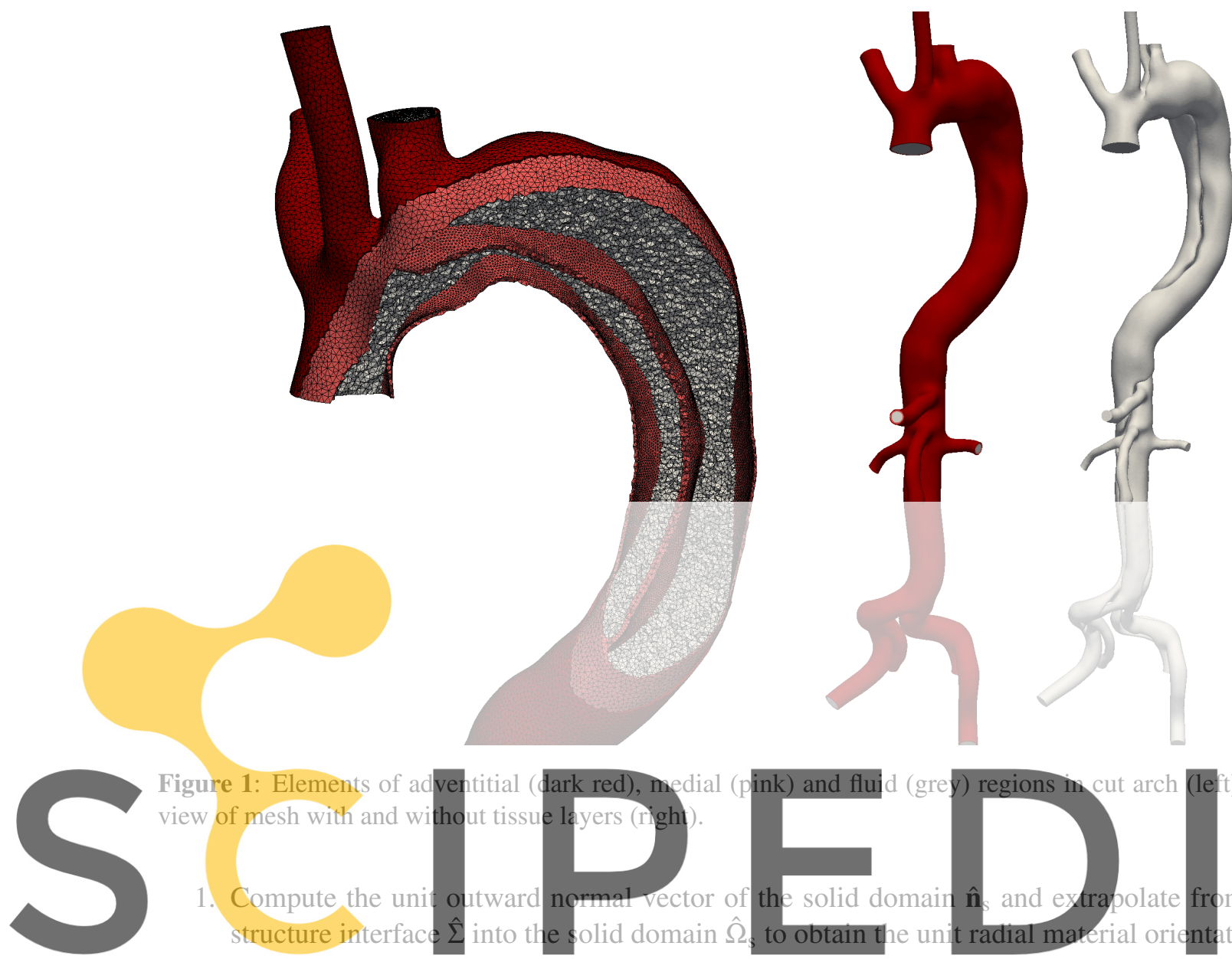

Figure 1: Elements of adventitial (dark red), medial (pink) and flu view of mesh with and without tissue
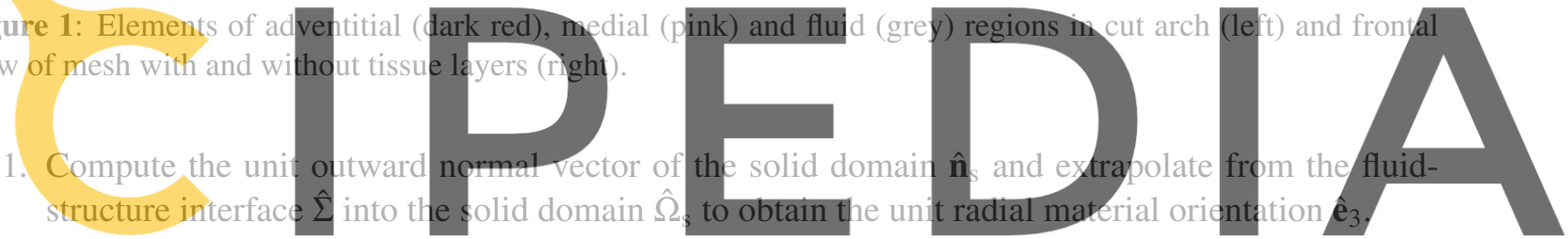

2. Solve the Laplace equation

Register for free at https//www.scipedia.çom to download the version without the watermark $-\vec{\nabla} \cdot \nabla \varphi=0$ in $\hat{\Omega}_{\mathrm{s}}$ with $\hat{\mathbf{n}} \cdot \nabla \varphi=0$ on $\hat{\Gamma}_{\mathrm{s}, \mathrm{e}} \cup \mathcal{\Sigma}$ and $\varphi=\bar{\varphi}$ on $\hat{\Gamma}_{\mathrm{s}, \mathrm{i}} \cup \hat{\Gamma}_{\mathrm{s}, \mathrm{o}}$,

with suitably chosen Dirichlet data $\bar{\varphi}$ on the in- and outlet faces of the solid domain, $\hat{\Gamma}_{\mathrm{s}, \mathrm{i}}$ and $\hat{\Gamma}_{\mathrm{s}, \mathrm{o}}$, and homogeneous Neumann conditions on $\hat{\Sigma}$ and the external boundary $\hat{\Gamma}_{\mathrm{e}}$.

3. Rotate the normalized flux vector $\hat{\mathbf{q}}=\hat{\nabla} \varphi /\|\hat{\nabla} \varphi\|_{2}$ in the plane spanned by $\hat{\mathbf{q}}$ and $\hat{\mathbf{n}}_{\mathrm{f}}$ by a minimal angle, such that $\hat{\mathbf{n}}_{\mathbf{f}} \cdot \hat{\mathbf{q}}=0$, i.e., they are perpendicular, resulting in the unit longitudinal vector $\hat{\mathbf{e}}_{2}$.

4. Compute the unit vector in circumferential direction $\hat{\mathbf{e}}_{1}$ orthogonal to $\hat{\mathbf{q}}$ and $\hat{\mathbf{n}}_{\mathrm{f}}$ using $\hat{\mathbf{e}}_{1}=\hat{\mathbf{q}} \times \hat{\mathbf{n}}_{\mathrm{f}}$.

The final outcome features natural transitions of longitudinal and circumferential vectors also close to bifurcations and in the flap region due to the smoothing properties of the Laplace operator and at the same time normal vectors of high quality are ensured. Due to the symmetric distribution of collagen fibres around the mean directions $\mathbf{M}_{i}$, the radial unit vector $\hat{\mathbf{e}}_{3}$ in the flap region needs to be extrapolated from one adjacent neighbor only to result in admissible local coordinate systems as showcased in Figure 2. 


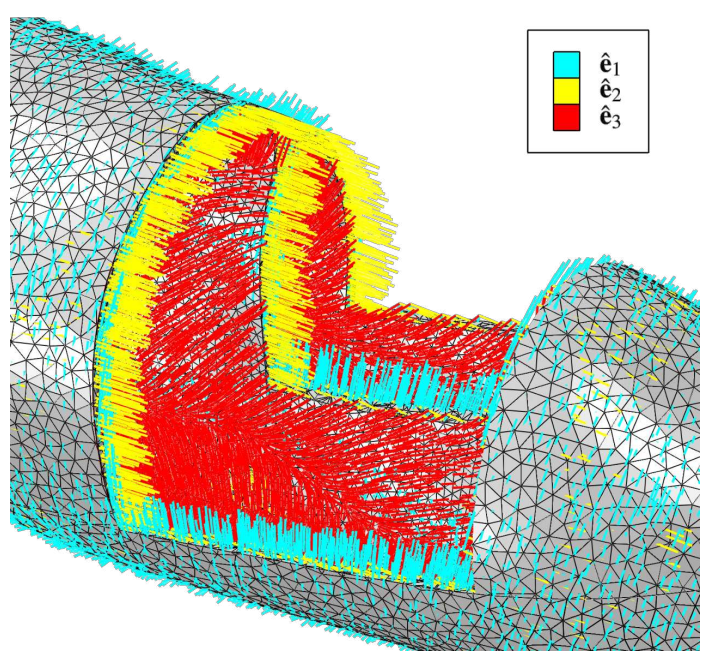

Figure 2: View cutting the dissected descending aortic arch: element-local coordinate systems for fibre orientation.

\subsection{Tissue prestress}

Another major modeling aspect of the cardiovascular system stems from the fact that the initial configuration as reconstructed from medical image data represents an equilibrium configuration of tissue deformation and blood flow. At the time of image acquisition, the blood flow already exerts some load

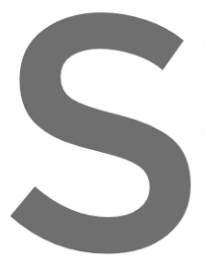

onto the vessel wall. This result in

when considering for nonito

under diastolic flow con

1. Compute the steady enforcing $\mathrm{d}_{\mathrm{S}}=0$,
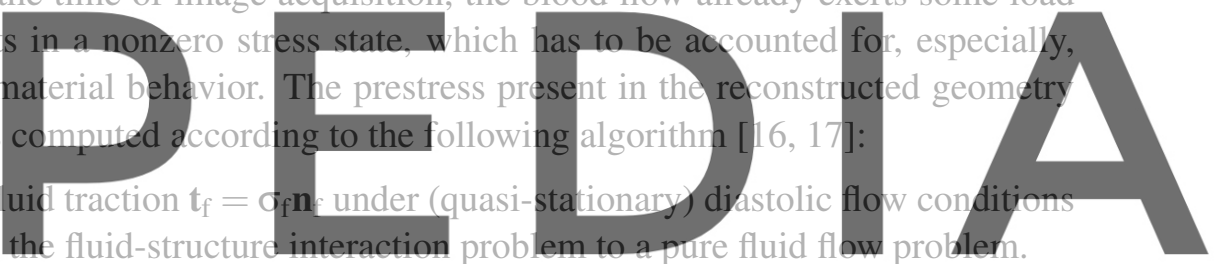

2. Until the convergence criterion. ||$d_{s} \|_{2}<\varepsilon$ is fulfilled, update the prestress $\mathbb{S}_{0}^{n}$ in the stationary

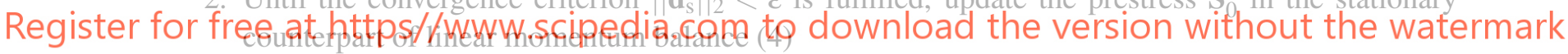

$$
-\hat{\nabla} \cdot\left[\mathbf{F}\left(\mathbf{S}+\mathbf{S}_{0}^{n}\right)\right]=0 \text { in } \hat{\Omega}_{\mathrm{s}} \quad \text { with } \quad \mathbf{F}\left(\mathbf{S}+\mathbf{S}_{0}^{n}\right) \hat{\mathbf{n}}_{\mathrm{s}}=\mathbf{t}_{\mathrm{f}} \text { on } \hat{\Sigma}
$$

and suitable boundary conditions on $\partial \hat{\Omega}_{\mathrm{s}} \backslash \hat{\Sigma}$, discussed in Section 2.5 .

As a consequence, the computed prestress tensor $\mathbf{S}_{0}$ counteracts the diastolic fluid load as convergence is reached, resulting in $\mathbf{d}_{\mathrm{s}} \approx \mathbf{0}$, i.e., negligible displacement, as can be seen in Figure 3.

\subsection{Boundary and coupling conditions}

Boundary conditions play a significant role in cardiovascular modeling, influencing relevant quantities of clinical interest and have thus been the focus of several studies including, e.g., [8, 18]. Within this contribution, the balance of linear momentum is supplied with homogenious Dirichlet boundary conditions on the in- and outlet boundaries of the aortic wall,

$$
\hat{\mathbf{d}}_{\mathrm{s}}=\mathbf{0} \text { on } \hat{\Gamma}_{\mathrm{s}, \mathrm{i}} \cup \hat{\Gamma}_{\mathrm{s}, \mathrm{o}}
$$



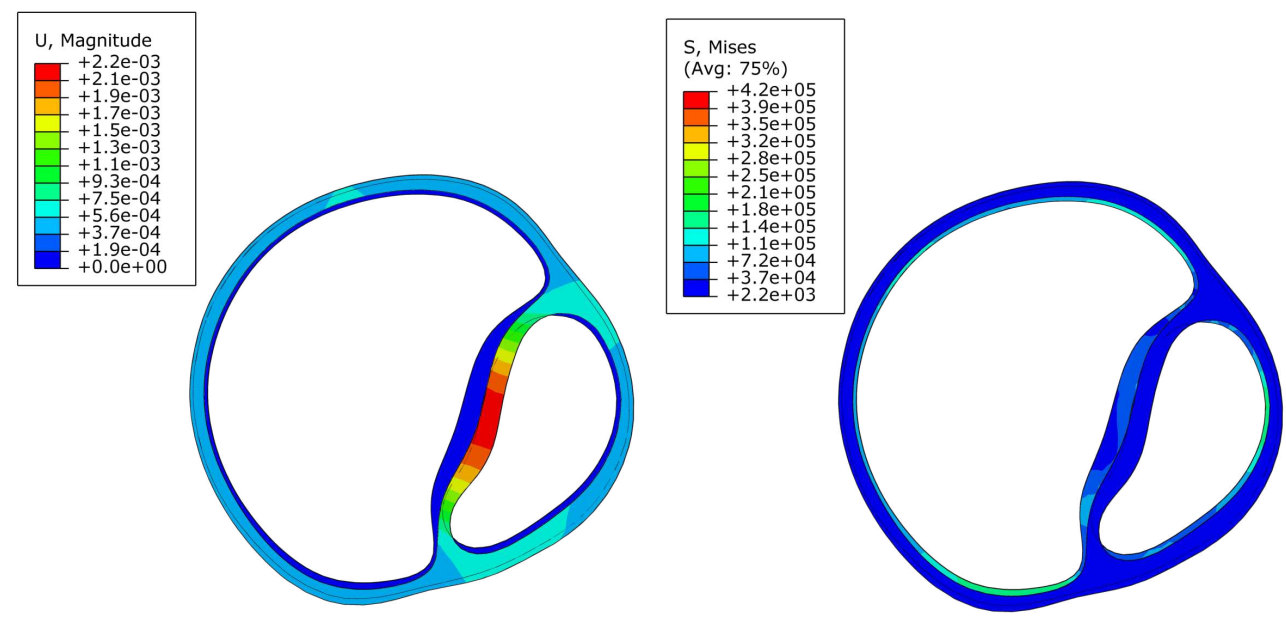

Figure 3: Initial and prestressed configurations of a selected cross-section: deformation in $\mathrm{m}$ (left) and Von Mises stress criterion in $\mathrm{Pa}$ (right). The converged prestress $\mathbf{S}_{0}$ compensates displacements due to diastolic fluid tractions.

effectively pinning the branch vessels and inlet cross-section in place at the boundary of the domain of interest and Robin boundary conditions of the form [18]
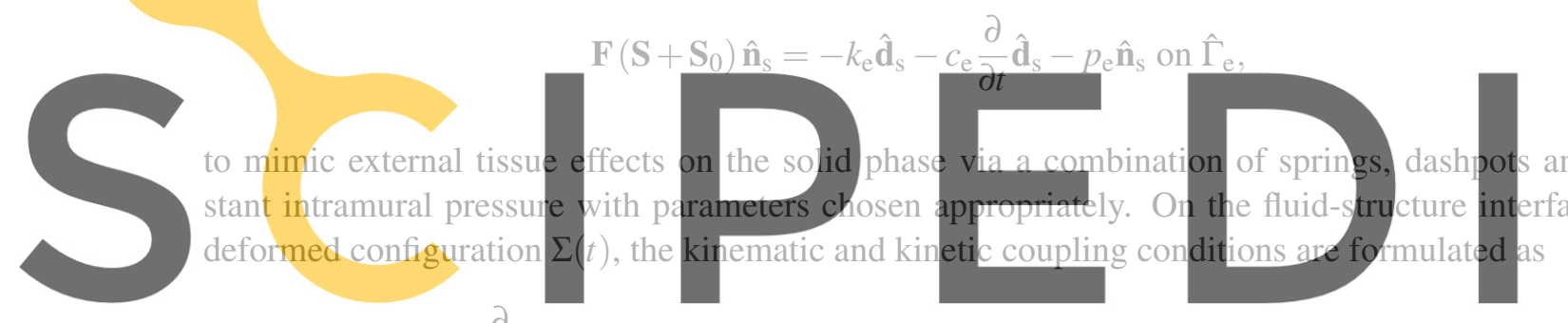

$$
\frac{\partial}{\partial} \hat{\mathbf{d}}_{\mathrm{S}}=\mathbf{v} \text { on } \Sigma(t) \text { and } \quad \sigma_{\mathrm{f}} \mathbf{n}_{\mathrm{f}}=J^{-1} \mathbf{F}\left(\mathbf{S}+\mathbf{S}_{0}\right) \mathbf{F}^{\mathrm{T}} \mathbf{n}_{\mathrm{f}} \text { on } \Sigma(t) .
$$

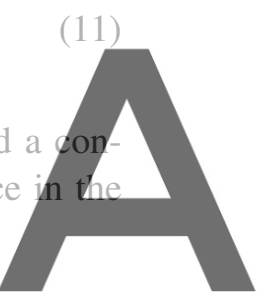

Register for free at https //www.scipedia.com to download the version without the watermark

Additionally, the geometric coupling condition

$$
\hat{\mathbf{d}}_{\mathrm{s}}=\hat{\mathbf{d}}_{\mathrm{f}} \text { on } \hat{\Sigma}
$$

ensures matching interface positions of fluid and solid phase at all times.

On the remaining boundaries, patient-specific flow data has to be taken into account. For this purpose, volumetric flow data as provided by [13] with mean velocities depicted in Figure 4 is used to enforce fluid velocity profiles on all in- and outlets. This choice is motivated by increased robustness using the current implementation when comparing it to standard 3-element Windkessel models [19, 20] to match flow splits to given data. Nonetheless, prescribing velocity profiles on in- and outlets via Dirichlet conditions such as

$$
\mathbf{v}=\overline{\mathbf{v}} \text { on } \Gamma_{\mathrm{f}, \mathrm{i}} \cup \Gamma_{\mathrm{f}, \mathrm{o}}
$$

is also admissible, noting that the focus of this study is on the solid constitutive modeling. 


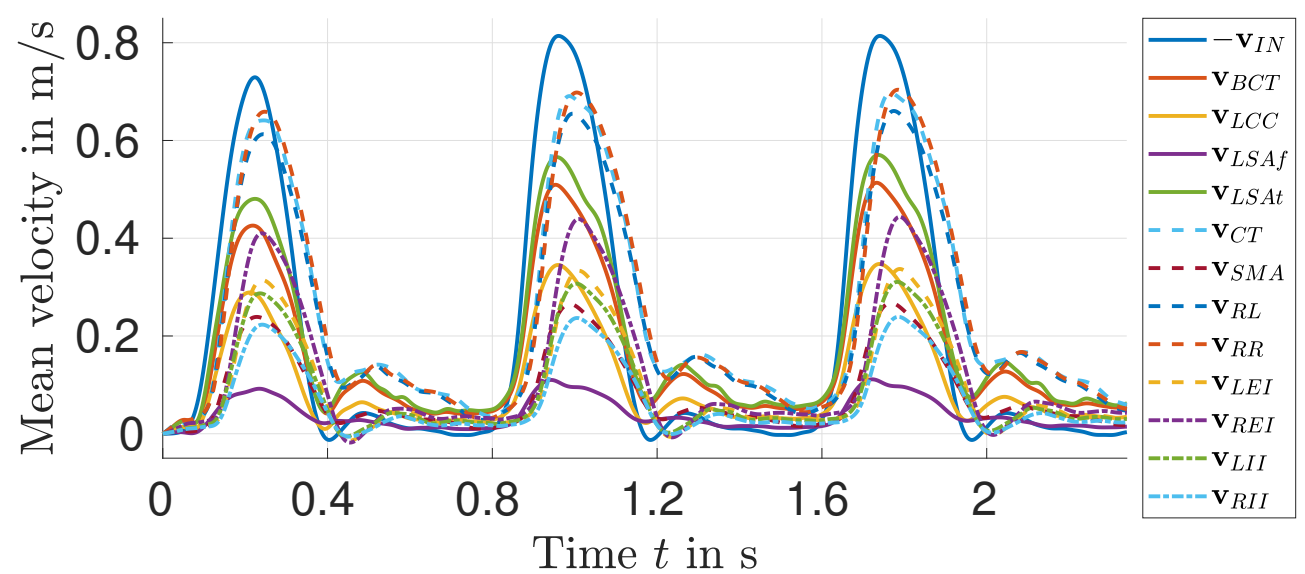

Figure 4: Mean velocities computed from flow data provided by [13].

\section{COMPUTATIONAL RESULTS}

In this section, we report first results obtained with the presented methodology. All of the above introduced algorithmic aspects have been included in a work-stream, which finally allows the numerical simulation of realistic aortic dissection datasets. However, as the case for any study dealing with patientspecific data, the set of parameters not identifiable in clinical practice increases with complexity. The patient cohorts and/or tiss ing to Table 1. In the fully c $0.78 \mathrm{~s}$ are carried out. Owint tear, the blood stream is found to be entering the $\mathrm{f}$ region. However, the naibrity of the blood strean
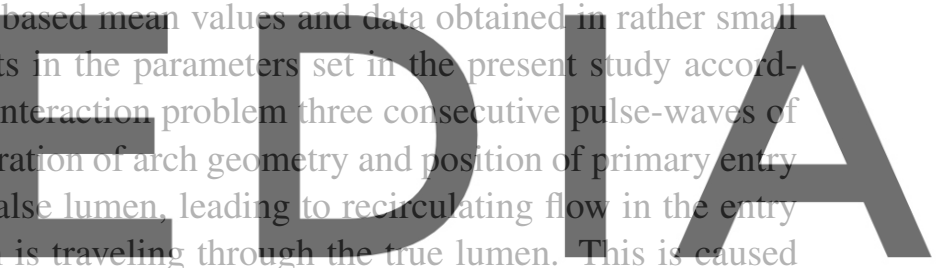

by the false lumen having re-entry tears only at the level of the iliac bifurcations and of course by the

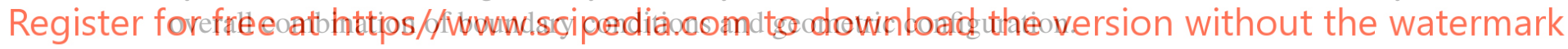

A maximum flap displacement of $2.8 \mathrm{~mm}$ is observed in the arch region at time $t=2.0 \mathrm{~s}$ as depicted together with the accompanying velocity at selected cross-sections in Figure 5. The complex flap motion is a consequence of the pressure difference in true and false lumina, recirculating flow and flap geometry.

\section{CONCLUSION}

Within this contribution, several important modeling aspects in the context of numerical modeling patientspecific aortic dissection were discussed. A computational domain was constructed based on medical image data, resulting in a multi-layered vessel wall. The tissue was considered as quasi-incompressible hyperelastic, fibre-reinforced continuum, additionally incorporating prestress present at the time of CTA acquisition. The setup of this computational pipeline now allows studying the solution's sensitivity to a change in the included parameters such as shear modulus, fluid viscosity, prescribed boundary conditions and more. At this current state, data extraction from the computational model postprocessing the results for validation with clinical data is needed. So, ongoing work focuses on (i) validation of the computational model and then (ii) comparing different tissue models of varying complexity to highlight 
Table 1: Material parameters taken from [13, 21-24], values in brackets indicate individual parameters for elements in flap, medial and adventitia regions in the format (flap and media/adventitia).

\begin{tabular}{lll}
\hline fluid density & $\rho_{\mathrm{f}}$ & $1060 \mathrm{~kg} / \mathrm{m}^{3}$ \\
dynamic viscosity & $\mu_{\mathrm{f}}$ & $3.5 \mathrm{mPa} \mathrm{s}$ \\
\hline solid density & $\rho_{\mathrm{s}}$ & $1200 \mathrm{~kg} / \mathrm{m}^{3}$ \\
bulk modulus & $\kappa_{\mathrm{b}}$ & $100 \mathrm{kPa}$ \\
fibre parameters & $k_{1}$ & $1.4 \mathrm{kPa}$ \\
& $k_{2}$ & 22.1 \\
shear modulus & $\mu$ & $(120 / 150) \mathrm{kPa}$ \\
fibre direction & $\alpha$ & $( \pm 27.47 / \pm 52.88)^{\circ}$ \\
fibre dispersion & $\kappa$ & $(0.12 / 0.25)$ \\
\hline exterior tissue: stiffness & $k_{e}$ & $10^{6} \mathrm{~Pa} / \mathrm{m}$ \\
\multicolumn{1}{c}{ damping } & $c_{e}$ & $10^{7} \mathrm{~Pa} \mathrm{~s} / \mathrm{m}$ \\
intramural pressure & $p_{e}$ & $7.10^{3} \mathrm{~Pa}$ \\
\hline
\end{tabular}

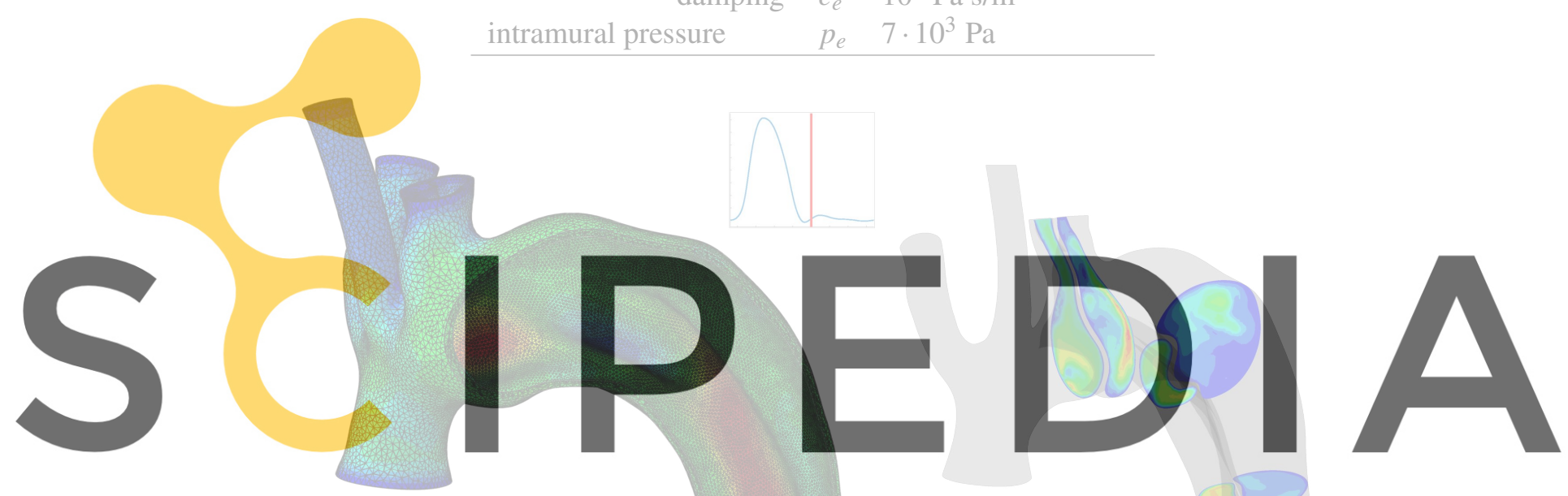

Register for free at https//www.scipedia.com to download the version without the watermark
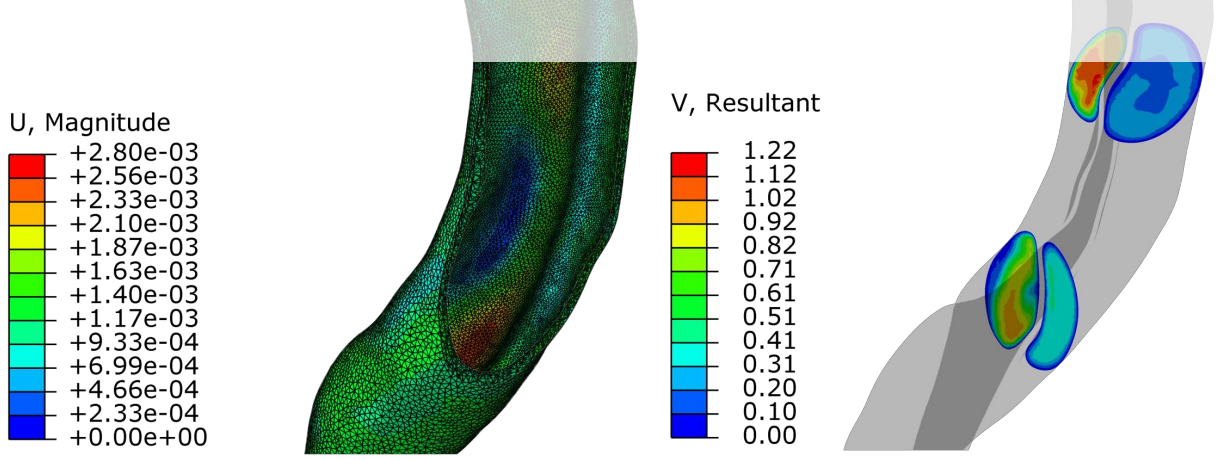

Figure 5: Solution in cut arch region: maximum deformation in $\mathrm{m}$ (left) and accompanying velocity in $\mathrm{m} / \mathrm{s}$ (right) in selected cross-sections at time $t=2.0 \mathrm{~s}$, as indicated by mean inlet velocity (top). 
the influence on dissection flap displacements and thereby a possibly substantial impact on the overall solution including parameters of clinical relevance such as TAWSS, OSI or true and false lumina pressure differences.

\section{ACKNOWLEDGEMENT}

The authors gratefully acknowledge Graz University of Technology for the financial support of the Leadproject Mechanics, Modeling and Simulation of Aortic Dissection.

\section{References}

[1] Z. Sun and T. Chaichana. A systematic review of computational fluid dynamics in type b aortic dissection. International Journal of Cardiology, 210:28-31, 2016.

[2] B.J. Doyle and P.E. Norman. Computational biomechanics in thoracic aortic dissection: Todays approaches and tomorrows opportunities. Annals of Biomedical Engineering, 44(1):71-83, 2016.

[3] J.J. Lee, G. D'Ancona, A. Amaducci, F. Follis, M. Pilato, and S. Pasta. Role of computational modeling in thoracic aortic pathology: A review. Journal of Cardiac Surgery, 29(5):653-662, 2014.

[4] B. Munshi, L.P. Parker, P.E. Norman, and B.J. Doyle. The application of computational modeling for risk prediction in type b aortic dissection. Journal of Vascular Surgery, 71(5):1789-1801, 2020.

[5] S. Sherifova and G.A. Holzapfel. Biomechanics of aortic wall failure with a focus on dissection and aneurysm: A review. Acta Biomaterialia, 99:1-17, 2019.

[6] W.N. Wan Ab Naim, P.B. Ganesan, Z. Sun, K.H. Chee, S.A. Hashim, and E. Lim. A perspective review on numerical simulations of hemodynamics in aortic dissection. The Scientific World Journal, 2014, 2014.

[7] A. Qiao, W. Yin, and B. Chu. Numerical simulation of fluidstructure interaction in bypassed debakey iii aortic dissection. Computer Methods in Biomechanics and Biomedical Engineering, 18 (11):1173-1180, 2015.

[8] M. Alimohammadi, J.M. Sherwood, M. Karimpour, O. Agu, S. Balabani, and V. Díaz-Zuccarini. Aortic dissection simulation models for clinical support: Fluid-structure interaction vs. rigid wall models. BioMedical Engineering Online, 14(1), 2015.

[9] M. Bonfanti, S. Balabani, J.P. Greenwood, S. Puppala, S. Homer-Vanniasinkam, and V. DíazZuccarini. Computational tools for clinical support: A multi-scale compliant model for haemodynamic simulations in an aortic dissection based on multi-modal imaging data. Journal of the Royal Society Interface, 14(136), 2017.

[10] M. Bonfanti, S. Balabani, M. Alimohammadi, O. Agu, S. Homer-Vanniasinkam, and V. DíazZuccarini. A simplified method to account for wall motion in patient-specific blood flow simulations of aortic dissection: Comparison with fluid-structure interaction. Medical Engineering \& Physics, 58:72 - 79, 2018.

[11] M. Bonfanti, G. Franzetti, G. Maritati, S. Homer-Vanniasinkam, S. Balabani, and V. Díaz- 
Zuccarini. Patient-specific haemodynamic simulations of complex aortic dissections informed by commonly available clinical datasets. Medical Engineering and Physics, 71:45-55, 2019.

[12] Y. Qiao, Y. Zeng, Y. Ding, J. Fan, K. Luo, and T. Zhu. Numerical simulation of two-phase nonnewtonian blood flow with fluid-structure interaction in aortic dissection. Computer Methods in Biomechanics and Biomedical Engineering, 22(6):620-630, 2019.

[13] K. Bäumler, V. Vedula, A.M. Sailer, J. Seo, P. Chiu, G. Mistelbauer, F.P. Chan, M.P. Fischbein, A.L. Marsden, and D. Fleischmann. Fluidstructure interaction simulations of patient-specific aortic dissection. Biomechanics and Modeling in Mechanobiology, 19(5):1607-1628, 2020.

[14] T.C. Gasser, R.W. Ogden, and G.A. Holzapfel. Hyperelastic modelling of arterial layers with distributed collagen fibre orientations. Journal of the Royal Society Interface, 3(6):15-35, 2006.

[15] G.A. Holzapfel, R.W. Ogden, and S. Sherifova. On fibre dispersion modelling of soft biological tissues: A review. Proceedings of the Royal Society A: Mathematical, Physical and Engineering Sciences, 475(2224), 2019.

[16] M.-C. Hsu and Y. Bazilevs. Blood vessel tissue prestress modeling for vascular fluidstructure interaction simulation. Finite Elements in Analysis and Design, 47(6):593 - 599, 2011. The TwentySecond Annual Robert J. Melosh Competition.

[17] R. Schussnig, M. Rolf-Pissarczyk, G.A. Holzapfel, and T.P. Fries. Fluid-structure interaction simulations of aortic dissection. PAMM, 20(1):e202000125, 2021.

[18] P. Moireau, N. Xiao, M. Astorino, C.A. Figueroa, D. Chapelle, C.A. Taylor, and J.-F. Gerbeau. External tissue support and fluid-structure simulation in blood flows. Biomechanics and Modeling in Mechanobiology, 11(1-2):1-18, 2012. cited By 105.

[19] N. Westerhof, J.-W. Lankhaar, and B.E. Westerhof. The arterial windkessel. Medical and Biological Engineering and Computing, 47(2):131-141, 2009.

[20] M. Esmaily Moghadam, I.E. Vignon-Clementel, R. Figliola, and A.L. Marsden. A modular numerical method for implicit $0 \mathrm{~d} / 3 \mathrm{~d}$ coupling in cardiovascular finite element simulations. Journal of Computational Physics, 244:63-79, 2013.

[21] M. Rolf-Pissarczyk, K. Li, D. Fleischmann, and G.A. Holzapfel. A discrete approach for modeling degraded elastic fibers in aortic dissection. Computer Methods in Applied Mechanics and Engineering, 373, 2021.

[22] K. Tanishita and K. Yamamoto. Vascular engineering: New prospects of vascular medicine and biology with a multidiscipline approach. 2016.

[23] A.J. Schriefl, G. Zeindlinger, D.M. Pierce, P. Regitnig, and G.A. Holzapfel. Determination of the layer-specific distributed collagen fibre orientations in human thoracic and abdominal aortas and common iliac arteries. Journal of the Royal Society Interface, 9(71):1275-1286, 2012.

[24] D. Enea, F. Henson, S. Kew, J. Wardale, A. Getgood, R. Brooks, and N. Rushton. Extruded collagen fibres for tissue engineering applications: Effect of crosslinking method on mechanical and biological properties. Journal of Materials Science: Materials in Medicine, 22(6):1569-1578, 2011. 